\title{
GESTIÓN EDUCATIVA EN UNA UNIVERSIDAD PÚBLICA BAJO EL MARCO DE UNA AUDITORÍA ACADÉMICA
}

\author{
EDUCATION MANAGEMENT IN A PUBLIC UNIVERSITY UNDER THE FRAMEWORK \\ OF AN ACADEMIC AUDITING \\ Luz Morales Calle* \\ Unidad de Posgrado de la Facultad de Ciencias Contables \\ Universidad Nacional Mayor de San Marcos-UNMSM / Lima-Perú \\ [Recepción: Diciembre de 2014 / Conformidad: Enero 2015]
}

\section{RESUMEN}

La problemática actual que tiene la gestión educativa de la facultad de Ciencias Administrativas de una universidad pública en el marco de la auditoría académica, promueve una investigación de enfoque cualitativo de tipo descriptivo cuya muestra de estudio fue de 39 docentes y 305 alumnos pertenecientes a la facultad de Ciencias Económicas, Administrativas y Contables de la Universidad Nacional San Cristóbal de Huamanga, donde se utilizó como instrumento dos cuestionarios: uno, sobre auditoría académica compuesta de 18 ítems y tres dimensiones: Evaluación de la situación académica, acciones de control del OCI y carencia de una auditoría académica y otro, para medir la variable gestión administrativa compuesta de 35 ítems correspondientes a tres dimensiones: Gestión administrativa, gestión académica y deficiencias existentes en el área académica.

Se encontró que la ausencia de una evaluación de la situación académica influye negativamente en la gestión educativa, las debilidades existentes en la gestión educativa son por falta de sistemas de calidad, procesos de auditoría académica, las acciones del OCI contribuyen poco a la gestión educativa, al no implementarse una auditoría académica. Finalmente, las deficiencias existentes en el área académica son por falta de políticas, sistemas de aseguramiento de calidad y procesos para tomar de decisiones sobre mejora de la calidad académica; concluyéndose, que el diseño e implementación de una auditoría académica puede mejorar significativamente la gestión educativa de la facultad de Ciencias Administrativas de una universidad pública.

Palabras clave:

Auditoria académica; gestión educativa.

\begin{abstract}
The current problems of educational management in the Faculty of Management of a public university in the framework of academic auditing, promotes research of qualitative approach and descriptive whose study sample was 39 teachers and 305 students from the Economics Sciences Faculty, Management and Accounting of the National University San Cristobal de Huamanga, where two instruments were used: first, a survey on academic audit consists of 18 items and three dimensions- an evaluation of the academic situation, OCI control actions and lack of an academic auditing- and second to measure the administrative management variable consisting of 35 items corresponding to three dimensions -administrative management, academic management and deficiencies in the academic area.

It was found that the absence of an evaluation of the academic situation affects negatively on the educational management, the weaknesses in the education management systems are due to the lack of quality systems, academic audit processes; the actions of OCI contribute little to educational management, by not implementing an academic auditing. Finally, deficiencies in the academic area are because of lack of policies, quality assurance systems and processes for decisions making on improving the academic quality; concluding that the design and implementation of an academic audit can significantly improve the educational management of the Faculty of Administrative Sciences in a public university.
\end{abstract}

\section{Keywords:}

Academic auditing; educational management.

\footnotetext{
* Maestrísta de Auditoría con mención en Auditoría de Gestión y Control Gubernamental- UNMSM. Bachiller en Ciencias Económicas y Administrativas.Email:1moralitosc@gmail.com
} 


\section{INTRODUCCIÓN}

El objetivo básico de la gestión de calidad total en las universidades es lograr un proceso de mejora continua de la calidad académica, de manera que el servicio académico recibido por los estudiantes se encuentre constantemente en correctas condiciones para que los estudiantes sean competitivos. Además, de mejorar todos los procesos internos, de forma tal de producir los servicios académicos sin defectos, mejorar todos los procesos y procedimientos internos de la universidad, la atención a los estudiantes, autoridades universitarias y docentes, el cumplimiento de la currícula en los tiempos establecidos y en los factores señalados que posibilite la acreditación académica o verificación de la gestión y calidad académica.

Sin embargo, la consecución de los objetivos académicos de la Universidad Nacional San Cristóbal de Huamanga (UNSCH) son parte de las debilidades encontradas en el análisis de los factores internos en el proceso de su planeamiento estratégico, debilidades referidas a deficiencia en la formulación de la currícula y planes de estudios, lenta incorporación a los avances científicos y tecnológicos, limitada implementación y uso de equipamiento de laboratorio y oficinas, escasas actividades de proyección y extensión universitaria, bajo nivel de rendimiento académico de los estudiantes, entre otros. Estas debilidades de la UNSCH son reflejo de la magra gestión en detrimento de la calidad académica y que debe ser revertido a fin que la universidad cumpla su rol para el que fue fundada y sus productos sean competitivos frente a un mundo laboral y académico altamente competitivo. Por ello, la gestión y calidad académica de la UNSCH deben ser verificadas, la cual deberá ser realizada por una instancia idónea como es el Órgano de Control Institucional a través de la Auditoría Académica, que es un sistema de gestión de control óptima y un aseguramiento de la calidad académica en las universidades; a la fecha, en la UNSCH no existe ninguna propuesta de normativa menos una aplicación de una auditoria académica.

La ausencia de técnicas, procedimientos y normas que permitan evaluar los componentes del control en el área académica de la UNSCH para determinar el grado de calidad de servicios prestados, limita fehacientemente un diagnóstico real de los servicios académicos que produce la UNSCH. Por ello, en base a lo expuesto, la presente investigación tiene como objeto proponer un modelo de auditoría académica que contribuya a verificar los aspectos constitutivos de la gestión y calidad académica y que con su análisis, conclusiones y recomendaciones mejore la gestión académica de la UNSCH.

\section{FUNDAMENTACIÓN DEL PROBLEMA}

El presente trabajo surge a razón que los actores en la gestión académica, nunca han sido evaluados por una auditoría académica; es decir, con la participación de personal profesional que tenga entrenamiento técnico, capacidad profesional y actitud independiente como auditores académicos. Por lo tanto los resultados de la presente investigación y la propuesta del modelo de auditoría académica serán aplicados en las universidades del país; así como, estarán en condiciones de incluir en la currícula de estudios una asignatura con la naturaleza y características de auditoría académica, como una herramienta básica para el proceso de evaluación para asegurar la calidad continua en beneficio, además, de otros agentes de la educación superior como los profesionales, asesoras y consultoras dedicadas a la auditoría integral o integrada, orientarán mejor sus acciones de estudio, evaluación y control interno.

Los resultados tienen un impacto profesional, social, institucional y empresarial; puesto que los beneficios y bondades del estudio pueden ser aprovechados por los centros educativos de nivel básico y superior universitario para asegurar su presencia en el mercado, en cuanto a la calidad de la producción académica y servicios y a los auditores se les presenta un nuevo campo para prestar sus servicios profesionales y les permitirá también, el ahorro de tiempo y costos en el proceso de la auditoría, evitando riesgos de auditoría. Los colegios profesionales promoverán la aplicación y uso de esta herramienta valiosa en la gestión académica y gerencial en los seminarios y cursos de capacitación y especialización. 


\section{OBJETIVO GENERAL}

Estudiar la problemática actual que se tiene en la gestión educativa de la facultad de Ciencias Administrativas de una universidad pública en el marco de la auditoría académica.

\section{OBJETIVOS ESPECÍFICOS}

1. Analizar de qué manera la ausencia de una evaluación de la situación académica de la facultad de Ciencias Administrativas influye negativamente en la gestión educativa.

2. Evaluar las debilidades de la gestión educativa que tiene la facultad.

3. Revisar si el Órgano de Control Institucional, en sus acciones de control contribuye a la gestión educativa de la facultad.

4. Identificar las deficiencias existentes en el área académica de la facultad para superarlas con la implementación de una auditoría académica.

\section{METODOLOGÍA}

La metodología del presente estudio, en concordancia a los objetivos e hipótesis formuladas es una investigación de nivel descriptivo, de tipo aplicada que busca sustentar, justificar y fundamentar la pertinencia del diseño e implementación de la auditoría académica dentro de las acciones de control del Órgano de Control Institucional - OCI de la Universidad Nacional San Cristóbal de Huamanga para medir la gestión y calidad académica.

En esta investigación que corresponde a un diseño no experimental y transversal, se sustentó en los métodos deductivo, inductivo y método hipotético deductivo, así como para la recopilación de la información se diseñaron dos instrumentos una para cada variable de estudio de acuerdo a la operacionalización de las variables; donde se ha identificado problemas en la gestión educativa. Así mismo, se está planteando la solución a través de una propuesta de un modelo de auditoría académica teniendo en cuenta la hipótesis; así como, los objetivos de la investigación estableciendo una relación directa entre los tres elementos metodológicos, finalizando con la contrastación de los objetivos específicos, lo que permitió consolidar el objetivo principal de la investigación. Los objetivos específicos contrastados fueron la base para emitir las conclusiones parciales de la investigación y constituirá el resultado del estudio que determinará la conclusión general del trabajo, que establecerá la interrelación entre el objetivo general y la conclusión general para contrastar la hipótesis general de la investigación, sobre la base del planteamiento metodológico y teórico. La población del estudio está constituida por todos los docentes, y alumnos de la facultad de Ciencias Económicas, Administrativas y Contables de la Universidad Nacional San Cristóbal de Huamanga (Ayacucho) que actualmente de acuerdo a los registros de la universidad es de 1475 estudiantes y 43 docentes que en total de población, suman 1518.

La muestra de un total 1475 estudiantes de la facultad de Ciencias Económicas, Administrativas y Contables de la Universidad Nacional de Huamanga (Ayacucho) se calculó aplicando la siguiente fórmula estadística:

$$
n=\frac{N^{*} Z_{a}^{2} p^{*} q}{d^{2 *}(N-1)+Z_{a}^{2 *} p^{*} q}
$$

\section{Donde}

$\mathrm{n}$ : Tamaño de muestra

$\mathrm{N}$ : Tamaño de la población

p: Es la proporción de la población que tiene la característica de interés: $50 \%$

q: Es la proporción de la población que no tiene la característica de interés: $50 \%$

z: Valor de la distribución normal estandarizada correspondiente al valor de confianza escogido 1.96

s: Error permisible, determina el investigador y representa que tan precisos se desean los resultados: $5 \%$

Reemplazando valores se tiene:

$$
\begin{aligned}
& \mathrm{n}=\frac{(1475)(1.96) 2(0.5)(0.5)}{(0.05) 2(1475-1)+(1.96) 2(0.5)(0.5)} \\
& \mathrm{n}=305
\end{aligned}
$$

La muestra de estudiantes estratificada es de 305.

La Muestra de un total de 43 docentes de la facultad de Ciencias Económicas, Administrativas y 
Contables de la Universidad Nacional San Cristobal de Huamanga (Ayacucho) se calculó aplicando la fórmula de poblaciones finitas:

$$
n=\frac{N^{*} Z_{a}^{2} p^{*} q}{d^{2 *}(N-1)+Z_{a}^{2} p^{*} q}
$$

Reemplazando valores se tiene:

$$
\begin{aligned}
& \mathrm{n}=\frac{(43)(1.96) 2(0.5)(0.5)}{(0.05) 2(43-1)+(1.96) 2(0.5)(0.5)} \\
& \mathrm{n}=39
\end{aligned}
$$

La muestra de docentes estratificada de 39.

Tabla No 01:

La Universidad Nacional San Cristóbal de Huamanga: Población-Resumen de Matrícula 2007

\begin{tabular}{|l|c|c|c|}
\hline \multicolumn{1}{|c|}{ FACULTADES } & DOCENTES & ESTUDIANTES & TOTAL \\
\hline Ciencias Agrarias & 65 & 1248 & 1313 \\
\hline Ciencias Biológicas & 56 & 699 & 755 \\
\hline Ciencias de la Educación & 111 & 899 & 1,010 \\
\hline Cs. Ecas., Adtvas. y Contables & 43 & 1,475 & 1,518 \\
\hline Ciencias Sociales & 56 & 1,145 & 1,201 \\
\hline Derecho y Cs. Políticas & 21 & 626 & 647 \\
\hline Enfermería & 36 & 419 & 455 \\
\hline Ing. Minas, Geol. y Civil & 102 & 1,259 & 1,361 \\
\hline Ing. Química y metalurgia & 50 & 668 & 718 \\
\hline Obstetricia & 44 & 404 & 448 \\
\hline \multicolumn{1}{|c|}{ TOTAL } & 584 & 8,842 & 9,426 \\
\hline
\end{tabular}

Fuente: Rectorado - Oficina General de Planificación y Presupuesto - Oficina de Desarrollo Institucional - UNSCH (2008). Boletín Estadístico 2007-2008. pp. 109 y 134.

Tabla No 02:

Población Facultad de Ciencias Económicas, Administrativas y Contables

\begin{tabular}{|c|c|c|}
\hline FACULTAD SEGMENTADA & SUJETOS & N \\
\hline \multirow{3}{*}{ Ciencias Económicas, Administrativas y Contables } & Alumnos & 1475 \\
\cline { 2 - 3 } & Docentes & 43 \\
\cline { 2 - 3 } & Total & $\mathbf{1 5 1 8}$ \\
\hline
\end{tabular}

Fuente: Elaboración propia.

Tabla No 03:

Muestra de estudio por segmentos de la Facultad de Ciencias Económicas, Administrativas y Contables

\begin{tabular}{|c|c|c|}
\hline FACULTAD SEGMENTADA & SUJETOS & N \\
\hline \multirow{3}{*}{ Ciencias Económicas, Administrativas y Contables } & Alumnos & 305 \\
\cline { 2 - 3 } & Docentes & 39 \\
\cline { 2 - 3 } & Total & $\mathbf{3 4 4}$ \\
\hline
\end{tabular}

Fuente: Elaboración propia. 


\section{MARCO TEÓRICO}

Vallejo, J. (2010) estudió en España, sobre la "Gestión de la calidad en los procesos de enseñanza-aprendizaje", con el objetivo de especificar y concretar la forma de garantizar la calidad en los procesos de enseñanza-aprendizaje, analizando la determinación de los problemas que conlleva la implantación de un Sistema de Garantía de la Calidad en los Centros Universitarios, así como los efectos asociados a la resistencia al cambio y las dificultades existentes; llegando a las conclusiones que la organización de las enseñanzas universitarias, propuesta por el Ministerio de Educación y Ciencia (MEC) en su documento del 26 de septiembre del 2006, incorpora la garantía de calidad como elementos básicos que debe contener los planes de estudios.

Vázquez, C.(2007) de la Universidad de San Andrés, Argentina ha realizado un profundo estudio titulado "Análisis de los Procesos de Implementación de las Políticas de Evaluación Universitaria en tres universidades nacionales: Estudio de Casos" con el objetivo de indagar sobre el proceso de implementación de las políticas de evaluación universitaria diseñadas desde el Estado nacional. Se diseñó un estudio de casos de abordaje cualitativo de naturaleza descriptiva. Se eligieron tres universidades nacionales argentinas que fueron unas de las primeras en ser evaluadas por la CONEAU y que difieren en su tamaño y tradición, así como en sus contextos institucionales. Las fuentes primarias utilizadas fueron entrevistas semi-estructuradas. El autor llegó a las conclusiones de analizar la implementación de los procesos de autoevaluación y evaluación externa en cada una de las universidades, se observó que los distintos actores que participaron en estos, ya sea pertenecientes a la universidad o externos, operaron en base a sus posiciones en torno a cuestiones y conflictos locales.

Barrenetxea, M. (2005) ha desarrollado una investigación en Europa titulada "La evaluación externa de la educación superior: España, Reino Unido y Suecia" en el que analiza los rasgos principales de los distintos sistemas universitarios; se hace un estudio de los mecanismos de evaluación externa utilizados en cada uno de los países, incluyendo valoraciones que las propias agencias de evaluación y las institucio- nes educativas han hecho de los resultados obtenidos. El autor llegó a las conclusiones, que desde 1992 hasta 1998 en el Reino Unido funcionan dos sistemas de aseguramiento de la calidad: Una evaluación horizontal de la calidad: evaluación de la calidad de la enseñanza y revisión de materias. Una evaluación vertical para revisar los mecanismos de aseguramiento de la calidad de las universidades: auditoría de la calidad académica. Su principal tarea ha sido revisar si los procedimientos de revisión de la calidad funcionan adecuadamente y elaborar un informe con las recomendaciones sobre las áreas determinadas que requieren mayor atención. El gobierno británico establece claramente que el principal propósito de la evaluación externa de la calidad es la rendición de cuentas y supone que la rendición de cuentas llevará a mejorar la calidad.

Baca (2011) realiza un estudio titulado "La auditoría académica herramienta moderna para la gestión académica y gerencial de las universidades de Lima Metropolitana (2004-2008)". La presente tesis tiene como finalidad lograr de las universidades públicas y privadas de Lima Metropolitana sean eficientes en su gestión académica y gerencial; así mismo, cumplan cabalmente el rol que la Ley No 23733, Ley universitaria lo prescribe. Permitirá conocer la forma en que se lleva a cabo la gestión académica y gerencial de la Universidad, y en base a esto llevar a cabo la auditoría académica, herramienta que mejorará la eficiencia de estas. Para este análisis se utilizó la investigación - acción, apoyándose con encuestas que sirvieron para elaborar gráficos ilustrativos que apoyen la investigación. Finalmente, presenta conclusiones y recomendaciones del tema.

Espinoza (2007) hace un estudio sobre la "Auditoría de gestión académica y gerencial en las universidades de la Región Chavín 2002 - 2004”. Con el objetivo de caracterizar y determinar la calidad de gestión académica y gerencial y, la calidad de formación académico-profesional de los estudiantes del último ciclo o año de estudios de las universidades de la Región Chavín años 2002-2004, bajo el enfoque y la concepción global de la auditoría moderna o integrada, encaminada a determinar la naturaleza, características, alcances y objetivos de la auditoría de gestión académica y gerencial. Se diseñó un es- 
tudio descriptivo explicativo. La población estuvo comprendida por los docentes y los estudiantes del último ciclo o año de estudios de 4 universidades de la Región Chavín. La muestra estuvo compuesta por 79 docentes y 312 estudiantes de cuatro universidades a quienes se les aplicó un cuestionario. Los resultados concluyen que son latentes los problemas económicos, sociales y educativos, las deficiencias de gestión y de formación de los estudiantes, las deficiencias del sistema educativo universitario de la Región Chavín (Ancash). La conclusión es que las universidades de la Región Chavín, son deficientes en la gestión académica, por cuanto no se hace evaluación del currículo y sílabos de manera periódica, no se dispone de docentes de calidad y con experiencia; falta un sistema apropiado del proceso de admisión de estudiantes y de docentes; exiguas remuneraciones a la investigación y desarrollo de proyectos en las universidades públicas, entre otras.

Barreda (2007) publica los resultados de su estudio titulado "Calidad Académica y su relación con la Gestión Académica en la Facultad de Educación de la Universidad Nacional de Cajamarca”, con el objetivo de analizar y determinar el nivel de influencia de gestión académica en la calidad académica. Con este fin se diseñó un estudio descriptivo correlacional. La población de estudio está constituido por 567 estudiantes de la facultad de Educación de los cuales se encuentran en los ciclos III, V, VII, IX, así como 64 docentes, con diferentes características y condición laboral. Se aplicó una encuesta sobre gestión académica y calidad académica cuyos resultados concluyen en que existe una relación positiva considerable que permite señalar que a mayor eficiencia de la gestión académica, mayor nivel de calidad académica.

Medina, Montañez, Brito \& Arce (2010), realizan un estudio titulado "La auditoría académica y las acciones de control para la buena administración de la Universidad Nacional Santiago Antúnez de Mayolo 2007-2008", con el objetivo de establecer la forma cómo una auditoría académica y las acciones de control pueden contribuir a la buena administración de la Universidad Nacional “Santiago Antúnez de Mayolo". El tipo de investigación, de acuerdo al diseño de investigación es descriptivo y aplicado. La población de estudio estuvo integrada por 5722 personas de la
UNASAM, conformada por 359 docentes ordinarios (incluidas las autoridades), 114 docentes contratados y 5249 estudiantes. La muestra estuvo constituida por 360 sujetos: 23 docentes ordinarios, 07 docentes contratados, y 330 estudiantes, a quienes se les aplicó la encuesta y se realizaron las entrevistas personales a las autoridades, jefes de oficina. Jefe de la Oficina de Control Institucional y representantes estudiantiles, los cuales suman un total de 80 entrevistados. Los resultados señalan que existe una fuerte relación entre la buena administración y las políticas de personal en la UNASAM, teniendo como parámetro la auditoría académica. La conclusión se menciona a la realización de la auditoría académica y las acciones de control en la Universidad Nacional "Santiago Antúnez de Mayolo" sí contribuyen mediana y eficientemente a la buena administración de la institución universitaria. El resultado de la investigación revela que las políticas de personal y otras normas como criterios de auditoría académica contribuyen eficientemente en la gestión administrativa de la UNASAM.

Espinoza, C.M. (2010) realiza un estudio titulado "La Auditoría Académica para la apropiada gestión en una institución educativa de nivel superior", con el objetivo de establecer la forma de cómo la auditoría académica puede coadyuvar a la apropiada gestión en una institución educativa de nivel superior. La población de estudio estuvo representada por todos los funcionarios y docentes de auditoría de las universidades del país así como los funcionarios de la Contraloría General de Tacna, que en total sumaban 3,110. La muestra estuvo constituida por 20 sujetos: 15 funcionarios de universidades, 03 funcionarios de Contraloría General de Tacna, y 2 docentes de auditoría. Se diseñó un estudio básico, descriptivo y explicativo. Este trabajo se basó tanto en el análisis documentario y encuestas. La conclusiones, es que la auditoría académica es una actividad multidisciplinaria coadyuvadora en el proceso de evaluación de medidas adoptadas por las dependencias de una institución educativa de nivel superior, conducentes a la optimización de recursos humanos, tecnológicos y materiales, para alcanzar sus propias metas y objetivos propuestos en sus planes operativos, a fin de ser examinadas en el contexto de su correspondiente plan estratégico institucional. 
Hernández (2007) ha desarrollado una investigación titulada "La Auditoría Académica y las acciones de control para la Buena Administración de las Universidades Nacionales en el Perú”. Sostiene que la auditoría académica contribuye a la buena administración de las universidades nacionales en el Perú, por ello en su última conclusión señala que la auditoría académica evalúa las políticas y normas académicas, la capacitación del personal docente y no docente, la programación curricular y los sílabos, la evaluación llevada a cabo por el personal docente.

\section{La Auditoría Académica}

El término auditar es aceptado por la Real Academia Española (RAE) como: examinar la gestión económica de una entidad a fin de comprobar si se ajusta a lo establecido por ley o costumbre y tiene una connotación financiera, lo que queda mejor aclarado e implícito como auditoría contable.

En cuanto a la palabra "académico" si bien es de uso frecuente, también es frecuentemente mal utilizado o entendido y se refiere según la RAE a lo "perteneciente o relativo a centros oficiales de enseñanza" entendiendo que en el caso de las universidades abarcaría la educación, la investigación, la difusión, la extensión y la previsión social. Lo rescatable y aplicable del verbo auditar al área educativa es la idea de comprobar si una gestión se ajusta a lo establecido por la normatividad de una institución y si logra los objetivos propuestos así, hoy día una gran cantidad de países usan la frase auditoría académica dentro de sus funciones de aseguramiento de la calidad de los procesos educativos, de manera regular y sin connotaciones punitivas o peyorativas.

\section{Gestión Educativa}

El término "gestión" es hacer las cosas a través de otras personas y el término "educativo"; según Manes (2004) el gerenciamiento educativo, viene a ser "Proceso de conducción de una institución educativa por medio del ejercicio de un conjunto de habilidades directivas orientadas a planificar, organizar, coordinar y evaluar la gestión estratégica de aquellas actividades necesarias para alcanzar eficacia pedagógica, eficiencia administrativa, efectividad comunitaria y trascendencia cultural" (p.17).
Bolam (citado por Bush, 2007), define la gestión de la educación como una "función ejecutiva para llevar a cabo la política acordada”. Se distingue la gestión de liderazgo educativo que tiene "como centro la responsabilidad de la formulación de políticas y, en su caso, de organización transformación".

Bush (2007) sostiene que: "La gestión educativa tiene que estar articulada con el propósito o los objetivos de la educación. Éstos son objeto de continuo debate y desacuerdo, pero el principio de vincular las actividades de gestión y tareas a los fines y objetivos de la escuela o de la universidad sigue siendo vital. Estos propósitos proporcionan el sentido fundamental de la orientación que debe sustentar la gestión de las instituciones educativas. La gestión se orienta a la consecución de ciertos objetivos educativas" (p.32).

En resumen podemos decir que la Gestión Educativa es, el proceso de planificar, organizar, dirigir y controlar las actividades de una institución mediante la utilización de los recursos humanos y materiales con el fin de llevar a cabo con eficacia y eficiencia las funciones de docencia, actividades de extensión y la investigación.

\section{HIPÓTESIS PRINCIPAL}

El diseño e implementación de una auditoría académica puede mejorar significativamente la gestión educativa de la facultad de Ciencias Administrativas de una universidad pública.

\section{HIPÓTESIS ESPECÍFICAS}

1. La ausencia de una evaluación de la situación académica de la facultad de Ciencias Administrativas influye negativamente en la gestión educativa al desconocerse los problemas en los procesos y resultados de la gestión académica.

2. Las principales debilidades en la gestión educativa que tiene la facultad de Ciencias Administrativas son la falta de sistemas de calidad y de procesos de auditoría académica.

3. Las acciones de control del Órgano de control Institucional - OCI contribuyen poco a la gestión educativa de la facultad al no implementarse una auditoría académica. 
4. Las principales deficiencias existentes en el área académica de la facultad que se deben superar con la implementación de una auditoría académica son la falta de políticas y sistemas de aseguramiento de la calidad y los procesos para toma de decisiones sobre mejora de la calidad académica.

\section{RESULTADOS}

A continuación, se muestra los resultados del estudio de la investigación, a través de una estadística de pruebas para las hipótesis principales y específicas, considerando que:
Ho: El diseño e implementación de una auditoría académica No puede mejorar significativamente la gestión educativa de la facultad de Ciencias Administrativas de una universidad pública.

Hi: El diseño e implementación de una auditoria académica puede mejorar significativamente la gestión educativa de la facultad de Ciencias Administrativas de una universidad pública.

Nivel de significancia: 0.05 para nivel de confianza de $95 \%$

Criterios de decisión:

Si p-valor $>0.05$ aceptar Hipótesis nula Ho

Si p-valor $=<0.05$ rechazar Hipótesis nula Ho

Tabla No 04:

Considera que se debe mejorar la gestión educativa Vs. Considera necesario y urgente el diseño e implementación de una auditoría académica

\begin{tabular}{|l|c|c|r|r|r|r|r|r|r|r|r|}
\hline \multirow{2}{*}{$\begin{array}{l}\text { Considera necesario } \\
\text { y urgente el diseño e } \\
\text { implementación de una } \\
\text { auditoría académica }\end{array}$} & \multicolumn{6}{|c|}{ CONSIDERA QUE SE DEBE MEJORAR LA GESTIÓN EDUCATIVA } \\
\cline { 2 - 12 } & $\begin{array}{c}\text { Nada } \\
\text { Frencia }\end{array}$ & $\%$ & $\mathbf{N}$ & $\%$ & $\mathbf{N}$ & $\%$ & $\mathbf{N}$ & $\%$ & $\mathbf{N}$ & $\%$ \\
\hline Nada necesario & 7 & $88 \%$ & 3 & $17 \%$ & 0 & $0 \%$ & 0 & $0 \%$ & 10 & $3 \%$ \\
\hline Poco necesario & 1 & $13 \%$ & 9 & $50 \%$ & 0 & $0 \%$ & 0 & $0 \%$ & 10 & $3 \%$ \\
\hline Bastante necesario & 0 & $0 \%$ & 6 & $33 \%$ & 87 & $98 \%$ & 40 & $17 \%$ & 133 & $39 \%$ \\
\hline Muy necesario & 0 & $0 \%$ & 0 & $0 \%$ & 2 & $2 \%$ & 189 & $83 \%$ & 191 & $56 \%$ \\
\hline Total & 8 & $100 \%$ & 18 & $100 \%$ & 89 & $100 \%$ & 229 & 1,0 & 344 & $100 \%$ \\
\hline
\end{tabular}

Chi cuadrado $=567,231$ p_valor $=0.00$

Prueba de Chi Cuadrado

\begin{tabular}{|l|r|r|r|}
\hline & \multicolumn{1}{|c|}{ Valor } & \multicolumn{1}{c|}{ Gl } & \multicolumn{1}{|c|}{ Sig. asintótica (bilateral) } \\
\hline Chi-cuadrado de Pearson & $567,231^{\mathrm{a}}$ & 9 &, 000 \\
\hline Razón de verosimilitudes & 345,327 & 9 &, 000 \\
\hline Asociación lineal por lineal & 250,420 & 1 &, 000 \\
\hline $\mathrm{N}^{\circ}$ de casos válidos & 344 & & \\
\hline
\end{tabular}

Fuente: Elaboración propia

\section{Decisión:}

Debido a que se ha obtenido una $\mathrm{p} \_$valor $=0.00<0.05$ entonces se rechaza la hipótesis nula y se acepta la hipótesis alterna $\mathrm{H} 1$ : por lo que se concluye que el diseño e implementación de una auditoría académica puede mejorar significativamente la gestión educativa de la facultad de Ciencias Administrativas de una universidad pública. 
Tabla No 05:

Considera que se debe mejorar la gestión educativa Vs Ausencia de la evaluación de situación académica como factor negativo

\begin{tabular}{|l|c|c|r|r|r|r|r|r|r|r|r|}
\hline \multirow{2}{*}{$\begin{array}{l}\text { Ausencia de la evaluación } \\
\text { de situación académica } \\
\text { como factor negativo }\end{array}$} & \multicolumn{6}{|c|}{ CONSIDERA QUE SE DEBE MEJORAR LA GESTIÓN EDUCATIVA } \\
\cline { 2 - 13 } & $\begin{array}{c}\text { Frecuencia } \\
(\mathbf{N})\end{array}$ & $\mathbf{0}$ & $\mathbf{N}$ & $\mathbf{\%}$ & $\mathbf{N}$ & $\mathbf{\%}$ & $\mathbf{N}$ & $\mathbf{\%}$ & $\mathbf{N}$ & $\%$ \\
\hline Muy bajo & 8 & $100 \%$ & 18 & $100 \%$ & 42 & $47 \%$ & 0 & $0 \%$ & 68 & $20 \%$ \\
\hline Bajo & 0 & $0 \%$ & 0 & $0 \%$ & 29 & $33 \%$ & 0 & $0 \%$ & 29 & $8 \%$ \\
\hline Bastante & 0 & $0 \%$ & 0 & $0 \%$ & 18 & $20 \%$ & 69 & $30 \%$ & 87 & $25 \%$ \\
\hline Muchísimo & 0 & $0 \%$ & 0 & $0 \%$ & 0 & $0 \%$ & 160 & $70 \%$ & 160 & $47 \%$ \\
\hline Total & 8 & $100 \%$ & 18 & $100 \%$ & 89 & $100 \%$ & 229 & $100 \%$ & 344 & $100 \%$ \\
\hline
\end{tabular}

Chi cuadrado $\mathrm{X} 2=336.836$; $\mathrm{p} \_$valor $=0.000$

Prueba de Chi Cuadrado

\begin{tabular}{|l|r|r|r|}
\hline & \multicolumn{1}{|c|}{ Valor } & \multicolumn{1}{c|}{ Gl } & \multicolumn{1}{|c|}{ Sig. asintótica (bilateral) } \\
\hline Chi-cuadrado de Pearson & $336,836^{\mathrm{a}}$ & 9 &, 000 \\
\hline Razón de verosimilitudes & 382,143 & 9 &, 000 \\
\hline Asociación lineal por lineal & 235,375 & 1 &, 000 \\
\hline $\mathrm{N}^{\circ}$ de casos válidos & 344 & & \\
\hline
\end{tabular}

Fuente: Elaboración propia

\section{Decisión:}

Debido a que se ha obtenido una p_valor $=0.00<0.05$ entonces se rechaza la hipótesis nula y se acepta la hipótesis alterna $\mathrm{H} 1$ : por lo que se concluye que la ausencia de una evaluación de la situación académica de la facultad de Ciencias Administrativas influye negativamente en la gestión educativa al desconocerse los problemas en los procesos y resultados de la gestión académica.

Tabla No 06:

Considera que se debe mejorar la gestión educativa Vs. En la facultad se cuenta con sistemas eficaces que garantizan la calidad académica

\begin{tabular}{|c|c|c|c|c|c|c|c|c|c|c|}
\hline \multirow{3}{*}{$\begin{array}{l}\text { En la facultad se cuenta } \\
\text { con sistemas eficaces } \\
\text { que garantizan la calidad } \\
\text { académica }\end{array}$} & \multicolumn{10}{|c|}{ CONSIDERA QUE SE DEBE MEJORAR LA GESTIÓN EDUCATIVA } \\
\hline & \multicolumn{2}{|c|}{ Nada } & \multicolumn{2}{|c|}{ Poco } & \multicolumn{2}{|c|}{ Bastante } & \multicolumn{2}{|c|}{ Muchísimo } & \multicolumn{2}{|c|}{ Total } \\
\hline & $\begin{array}{l}\text { Frecuencia } \\
(\mathbf{N})\end{array}$ & $\%$ & $\mathbf{N}$ & $\%$ & $\mathbf{N}$ & $\%$ & $\mathbf{N}$ & $\%$ & $\mathbf{N}$ & $\%$ \\
\hline Nunca & 8 & $100 \%$ & 18 & $100 \%$ & 89 & $100 \%$ & 135 & $59 \%$ & 250 & $73 \%$ \\
\hline A veces & 0 & $0 \%$ & 0 & $0 \%$ & 0 & $0 \%$ & 43 & $19 \%$ & 43 & $13 \%$ \\
\hline Con frecuencia & 0 & $0 \%$ & 0 & $0 \%$ & 0 & $0 \%$ & 29 & $13 \%$ & 29 & $8 \%$ \\
\hline Siempre & 0 & $0 \%$ & 0 & $0 \%$ & 0 & $0 \%$ & 22 & $10 \%$ & 22 & $6 \%$ \\
\hline Total & 8 & $100 \%$ & 18 & $100 \%$ & 89 & $100 \%$ & 229 & $100 \%$ & 344 & $100 \%$ \\
\hline
\end{tabular}


Chi cuadrado X $2=64.954$ p_valor $=0.00<0.05$

\section{Prueba de Chi Cuadrado}

\begin{tabular}{|l|r|r|r|}
\hline & \multicolumn{1}{|c|}{ Valor } & \multicolumn{1}{c|}{ Gl } & \multicolumn{1}{c|}{ Sig. asintótica (bilateral) } \\
\hline Chi-cuadrado de Pearson & $64.954^{\mathrm{a}}$ & 9 &, 000 \\
\hline Razón de verosimilitudes & 93,411 & 9 &, 000 \\
\hline Asociación lineal por lineal & 38,609 & 1 &, 000 \\
\hline $\mathrm{N}^{\circ}$ de casos válidos & 344 & & \\
\hline
\end{tabular}

Fuente: Elaboración propia

\section{Decisión:}

Debido a que se ha obtenido una $\mathrm{p} \_$valor $=0.00<0.05$ entonces se rechaza la hipótesis nula y se acepta la hipótesis alterna $\mathrm{H} 1$ : por lo que se concluye que las principales debilidades en la gestión educativa que tiene la facultad de Ciencias Administrativas son la falta de sistemas de calidad y de procesos de auditoría académica.

Tabla No 07:

Considera que se debe mejorar la gestión educativa Vs. El Órgano de Control Institucional (OCI) tiene la capacidad funcional

\begin{tabular}{|c|c|c|c|c|c|c|c|c|c|c|}
\hline \multirow{3}{*}{$\begin{array}{l}\text { El Órgano de Control Institucional (OCI) } \\
\text { tiene la capacidad funcional para manejar } \\
\text { un sistema de auditoría académica como } \\
\text { un sistema de gestión de control ópti- } \\
\text { mo para un aseguramiento de la calidad } \\
\text { académica. }\end{array}$} & \multicolumn{10}{|c|}{$\begin{array}{l}\text { CONSIDERA QUE SE DEBE MEJORAR LA } \\
\text { GESTIÓN EDUCATIVA }\end{array}$} \\
\hline & \multicolumn{2}{|c|}{ Nada } & \multicolumn{2}{|c|}{ Poco } & \multicolumn{2}{|c|}{ Bastante } & \multicolumn{2}{|c|}{ Muchísimo } & \multicolumn{2}{|c|}{ Total } \\
\hline & $\mathbf{N}$ & $\%$ & $\mathbf{N}$ & $\%$ & $\mathbf{N}$ & $\%$ & $\mathbf{N}$ & $\%$ & $\mathbf{N}$ & $\%$ \\
\hline Ninguna & 8 & $100 \%$ & 18 & $100 \%$ & 84 & $94 \%$ & 80 & $35 \%$ & 190 & $55 \%$ \\
\hline Poca & 0 & $0 \%$ & 0 & $0 \%$ & 5 & $6 \%$ & 75 & $33 \%$ & 80 & $23 \%$ \\
\hline Bastante & 0 & $0 \%$ & 0 & $0 \%$ & 0 & $0 \%$ & 54 & $24 \%$ & 54 & $16 \%$ \\
\hline Muchísima & 0 & $0 \%$ & 0 & $0 \%$ & 0 & $0 \%$ & 20 & $9 \%$ & 20 & $6 \%$ \\
\hline Total & 8 & $100 \%$ & 18 & $100 \%$ & 89 & $100 \%$ & 229 & $100 \%$ & 344 & $100 \%$ \\
\hline
\end{tabular}

Chi cuadrado $\mathrm{X}^{2}=115.205$, $\mathrm{p}$ _valor $=0.00<0.05$

Prueba de Chi Cuadrado

\begin{tabular}{|l|r|r|r|}
\hline & \multicolumn{1}{|c|}{ Valor } & \multicolumn{1}{c|}{ Gl } & \multicolumn{1}{|c|}{ Sig. asintótica (bilateral) } \\
\hline Chi-cuadrado de Pearson & $115,205^{\mathrm{a}}$ & 9 &, 000 \\
\hline Razón de verosimilitudes & 144,965 & 9 &, 000 \\
\hline Asociación lineal por lineal & 71,860 & 1 &, 000 \\
\hline $\mathrm{N}^{\circ}$ de casos válidos & 344 & & \\
\hline
\end{tabular}

Fuente: Elaboración propia

\section{Decisión:}

Debido a que se ha obtenido una $\mathrm{p} \_$valor $=0.00<0.05$ entonces se rechaza la hipótesis nula y se acepta la hipótesis alterna $\mathrm{H} 1$ : por lo que se concluye que las acciones de control de la Oficina de Control Interno contribuyen poco a la gestión educativa de la facultad al no implementarse una auditoría académica. 
Tabla No 08:

Considera que se debe mejorar la gestión educativa Vs. Se diseñó o propuso un plan

\begin{tabular}{|c|c|c|c|c|c|c|c|c|c|c|}
\hline \multirow{3}{*}{$\begin{array}{l}\text { Se diseñó o propuso un plan para ase- } \\
\text { gurar que los procesos departamentales, } \\
\text { políticas y procedimientos influyan } \\
\text { positivamente en las actividades. }\end{array}$} & \multicolumn{10}{|c|}{$\begin{array}{l}\text { CONSIDERA QUE SE DEBE MEJORAR LA } \\
\text { GESTIÓN EDUCATIVA }\end{array}$} \\
\hline & \multicolumn{2}{|c|}{ Nada } & \multicolumn{2}{|c|}{ Poco } & \multicolumn{2}{|c|}{ Bastante } & \multicolumn{2}{|c|}{ Muchísimo } & \multicolumn{2}{|c|}{ Total } \\
\hline & $\mathbf{N}$ & $\%$ & $\mathbf{N}$ & $\%$ & $\mathbf{N}$ & $\%$ & $\mathbf{N}$ & $\%$ & $\mathbf{N}$ & $\%$ \\
\hline Nada cierto & 8 & $100 \%$ & 18 & $100 \%$ & 87 & $98 \%$ & 126 & $55 \%$ & 239 & $69 \%$ \\
\hline Poco cierto & 0 & $0 \%$ & 0 & $0 \%$ & 2 & $2 \%$ & 82 & $36 \%$ & 84 & $24 \%$ \\
\hline Cierto & 0 & $0 \%$ & 0 & $0 \%$ & 0 & $0 \%$ & 14 & $6 \%$ & 14 & $4 \%$ \\
\hline Muy cierto & 0 & $0 \%$ & 0 & $0 \%$ & 0 & $0 \%$ & 7 & $3 \%$ & 7 & $2 \%$ \\
\hline Total & 8 & $100 \%$ & 18 & $100 \%$ & 89 & $100 \%$ & 229 & $100 \%$ & 344 & $100 \%$ \\
\hline
\end{tabular}

Chi cuadrado $X^{2}=67.592$ p_valor $=0.00<0.05$

Prueba de Chi Cuadrado

\begin{tabular}{|l|r|r|r|}
\hline & \multicolumn{1}{|c|}{ Valor } & \multicolumn{1}{c|}{ Gl } & Sig. asintótica (bilateral) \\
\hline Chi-cuadrado de Pearson & $67,592^{\mathrm{a}}$ & 9 &, 000 \\
\hline Razón de verosimilitudes & 89,898 & 9 &, 000 \\
\hline Asociación lineal por lineal & 41,536 & 1 &, 000 \\
\hline N de casos válidos & 344 & & \\
\hline
\end{tabular}

Fuente: Elaboración propia

\section{Decisión:}

Debido a que se ha obtenido una $\mathrm{p} \_$valor $=0.00$ $<0.05$ entonces se rechaza la hipótesis nula y se acepta la hipótesis alterna $\mathrm{H} 1$ : por lo que se concluye que las principales deficiencias existentes en el área académica de la facultad que se deben superar con la implementación de una auditoría académica son la falta de políticas y sistemas de aseguramiento de la calidad y los procesos para toma de decisiones sobre mejora de la calidad académica.

La presente investigación se propuso analizar la problemática actual en la gestión educativa de la facultad de Ciencias Administrativas de una universidad pública en el marco de la auditoría académica, por lo que se aplicó dos encuestas a una muestra de docentes y de estudiantes para conocer cómo perciben el problema en sus dos variables: Auditoría académica y Gestión educativa.

Se ha hallado que la ausencia de una evaluación de la situación académica de la facultad de Ciencias
Administrativas influye negativamente en la gestión educativa al desconocerse los problemas en los procesos y resultados de la gestión académica. Para determinar la significancia de este resultado se ha establecido una asociación entre la consideración de la ausencia de la evaluación de la situación académica como un factor negativo y el reconocimiento de la necesidad de mejorar las gestión educativa.

$(\mathrm{X} 2=336.83$; p_valor $=0.000)$.

Este resultado se puede explicar por el hecho de que los estudiantes y los docentes encuestados, reconocen, en su mayoría, que la ausencia de la evaluación de la situación académica de la facultad de Ciencias Administrativas está afectando seriamente la gestión educativa por cuanto limita un diagnóstico real de los servicios académicos que produce la facultad, no permite conocer cómo se lleva a cabo la gestión académica y gerencial de la facultad, no permite conocer las condiciones y circunstancias en que se desarrollan las actividades académicas, no permite 
conocer los resultados de la gestión académica, no permite hacer propuestas para mejorar la eficiencia de los procesos y actividades académicas, por lo que esta ausencia está afectando seriamente la calidad académica en la facultad.

Asimismo, se ha establecido que las principales debilidades en la gestión educativa que tiene la facultad de Ciencias Económicas, Administrativas y Contables son la falta de sistemas de calidad y de procesos de auditoría académica. Este resultado se evidencia al relacionar la necesidad de mejorar la gestión educativa y el reconocimiento de la falta de sistemas eficaces que garanticen la calidad académica (Chi cuadrado X $2=64.95$ P_valor $=0.00<0.05$ ).

Se ha hallado que las acciones de control de la Oficina de Control Interno contribuyen poco a la gestión educativa de la facultad al no implementarse una auditoría académica. Este supuesto se ha verificado al hallar una asociación significativa entre el reconocimiento de la necesidad de mejorar la gestión educativa en la facultad y el reconocimiento de que la Oficina de Control Interno (OCI) tiene la capacidad funcional para manejar un sistema de auditoría académica como un sistema de gestión de control óptimo para un aseguramiento de la calidad académica. (X 2 = 115.20 P_valor $=0.00<0.05)$

Finalmente, se ha verificado que las principales deficiencias existentes en el área académica de la facultad que se deben superar con la implementación de una auditoria académica son la falta de políticas y sistemas de aseguramiento de la calidad y los procesos para toma de decisiones sobre mejora de la calidad académica. Este hecho está relacionado a la asociación entre la necesidad de mejorar la gestión educativa y si la facultad diseñó o propuso un plan para asegurar que los procesos departamentales, políticos y procedimientos influyan positivamente en las actividades (X2 $=67.59$; $\mathrm{P}_{\text {_ }}$ valor $=0.00<0.05$ ).

Al relacionar ambas variables se ha verificado que el diseño e implementación de una auditoría académica puede mejorar significativamente la gestión educativa de la facultad de Ciencias Administrativas de una universidad pública. La verificación de esta hipótesis general se ha estimado mediante el test Chi cuadrado entre la necesidad de mejorar la gestión educativa y diseñar e implementar una auditoría académica en la facultad.

$($ Chi cuadrado $=567,231$ p_valor $=0.00)$.

Los datos recopilados de parte de los estudiantes y docentes de la facultad nos evidencian que en la actualidad, no existen sistemas de control que garanticen avanzar hacia el mejoramiento de la gestión y la calidad universitaria. Lo cual debe empezar con una política clara de las autoridades universitarias para hacer los cambios necesarios, para adoptar un sistema de mejoramiento de la calidad universitaria en base a la implementación de una auditoría académica.

\section{CONCLUSIONES}

1. El estudio de la problemática de la gestión educativa en el marco de la auditoría académica han revelado que la ausencia de evaluaciones de la situación académica está influyendo negativamente en la gestión de la facultad y por consiguiente en la calidad universitaria, por lo que la percepción de los docentes y estudiantes es que el diseño e implementación de una auditoría académica puede mejorar significativamente la gestión educativa de la facultad de Ciencias Administrativas de una universidad pública.

2. La ausencia de la evaluación de la situación académica de la facultad de Ciencias Administrativas está afectando seriamente la gestión educativa por cuanto limita un diagnostico real de los servicios académicos que produce la facultad, no permite conocer cómo se lleva a cabo la gestión académica y gerencial de la facultad, no permite conocer las condiciones y circunstancias en que se desarrollan las actividades académicas, no permite conocer los resultados de la gestión académica, no permite hacer propuestas para mejorar la eficiencia de los procesos y actividades académicas por lo que esta ausencia, está afectando seriamente la calidad académica en la facultad. En resumen, la ausencia de una evaluación de la situación académica de la facultad de Ciencias Administrativas influye negativamente en la gestión educativa al desconocerse los problemas en los procesos y resultados de la gestión académica. 
3. Se ha evidenciado que la Oficina de Control Interno, en sus acciones de control no contribuye a la gestión educativa de la facultad al no implementarse una auditoría académica a pesar de que tiene la capacidad funcional para hacerlo.

4. Las principales deficiencias existentes en el área académica de la facultad que se deben superar con el diseño e implementación de una auditoria académica son las faltas de políticas y sistemas de aseguramiento de la calidad y los procesos para toma de decisiones sobre mejora de la calidad académica. Es decir, que mientras en la facultad no asuma una política de aseguramiento de la calidad por parte de las autoridades, no se puede lograr una formación universitaria de calidad.

\section{RECOMENDACIONES}

1. Diseñar y proponer un sistema de evaluación interna de la gestión académica como parte de un sistema de auditoría académica orientada a mejorar significativamente la Gestión educativa de la facultad de Ciencias Económicas, Administrativas y Contables de la Universidad Nacional de San Cristóbal de Huamanga.

2. Diseñar sistemas de evaluación orientado a un diagnóstico real de los servicios académicos que produce la facultad, que permita conocer cómo se lleva a cabo la gestión académica y gerencial de la facultad, así como las condiciones y circunstancias en que se desarrollan las actividades académicas, conocer los resultados de la gestión académica, mejorar la eficiencia de los procesos y actividades académicas.

3. Extender la cobertura de la Oficina de Control Interno, para que contribuya más a la gestión educativa de la facultad, implementando una auditoría académica de forma periódica.

4. Promover políticas y sistemas de aseguramiento de la calidad y procesos para toma de decisiones sobre mejora de la calidad académica para lograr una formación universitaria de calidad, a través de una propuesta de un modelo de auditoría académica para la UNSCH.

\section{REFERENCIAS BIBLIOGRÁFICAS}

1. BACA, A. (2011). La auditoría académica herramienta moderna para la gestión académica y gerencial de las universidades de Lima Metropolitana (2004-2008). Tesis Maestría. Universidad Nacional Mayor de San Marcos.

2. BARREDA, I. (2007). Calidad académica y su relación con la gestión académica en la Facultad de Educación de la Universidad Nacional de Cajamarca. Tesis Maestría. Universidad Nacional Mayor de San Marcos.

3. BARRENETXEA, M., CARDONA, A., ECHEVARRÍA, C. (2005, Febrero) La evaluación externa de la educación superior: España, Reino Unido y Suecia. Ponencia presentada en el V Congreso Internacional Virtual de Educación.

4. BUSH, T. (2007). Theories of Educational Leadership and Management. Londres: SAGE Publications.

5. DEUPREE, L.; Johnson, M.E. y Lenn, M.P. (eds.) (2002). Ocde-us, Forum on Trade in Educational Services: Conference Proceedings, Washington, The Center for Quality Assurance in Higher Education.

6. HERNÁNDEZ, D. (2007, junio 6). Auditoría académica y control para la administración de las universidades nacionales en el Perú. Recuperado de http://www.gestiopolis.com/auditoria-academica-control-administracion-universidades-nacionales-peru/

7. ESPINOZA, A. (2007). Auditoría de gestión académica y gerencial en las universidades de la Región Chavín 2002 - 2004. Tesis Maestría. Universidad Nacional Mayor de San Marcos.

8. ESPINOZA, C. (2010). La auditoría académica para la apropiada gestión en una institución educativa de nivel superior. Tesis Universidad Privada de Tacna.

9. GÓMEZ, M. (2006). Introducción a la metodología de la investigación científica. Córdoba, Argentina: Editorial Brujas.

10. GUPTA, B. (2011). Academic Audit. Nueva Delhi, India: Concept Publishing Company. 
11. MANES, J. (2004) Gestión Estratégica para Instituciones Educativas. Santiago de Chile: Ediciones Granica S.A.

12.MEDINA, M., MONTANEZ, W., BRITO, E. \& ARCE, F. (julio/diciembre, 2010) La auditoría académica y las acciones de control para la buena administración de la Universidad Nacional Santiago Antúnez de Mayolo 2007-2008. Revista Aporte Santiaguino, 3(2), 185-190.
13.VALLEJO J. (2010) Gestión de la calidad en los procesos de enseñanza-aprendizaje (Tesis doctoral) Recuperado de http://riuma.uma.es/xmlui/ bitstream/handle/10630/4616/TDR_VALLEJO_GARCIA.pdf?sequence $=6$

14.VÁSQUEZ, C. (2007) Análisis de los Procesos de Implementación de las Políticas de Evaluación Universitaria en tres universidades nacionales: Estudio de Casos (Tesis doctoral) Recuperado de http://live.v1.udesa.edu.ar/files/MAEEDUCACION/SINTESISTESISCECILIA\%20 VAZQUEZ.PDF 